\title{
ANÁLISIS OCUPACIONAL Y DE GÉNERO EN LA INFANCIA: APLICACIÓN DE LOS MAPAS CORPORALES NARRADOS DESDE UN GRUPO DE DISCUSIÓN
}

\author{
Natalia Rivas-Quarneti' ${ }^{1}$, Rocío Ferreira-Marante ${ }^{2}$, Alicia Tojeiro-Ríos ${ }^{3}$ e Inés Viana-Moldes ${ }^{4}$ \\ Universidade da Coruña, España; ${ }^{1}$ natalia.rivas.quarneti@udc.es, ${ }^{2}$ rocio.ferreira.marante@gmail.com \\ 35beipargapondal@gmail.com, ${ }^{4}$ ines.viana@udc.es
}

\begin{abstract}
Resumen. El objetivo de esta investigación fue explorar las percepciones de alumnado de Educación Primaria de un colegio público de A Coruña (Galicia, España), sobre los imaginarios colectivos en relación con las ocupaciones, expresión de género y estereotipos hegemónicos. Se plantea una investigación secundaria, utilizando una experiencia socioeducativa previa basada en mapas corporales narrados, en la que un grupo de niñas y niños habían co-creado las historias de dos personajes, Verónica y Antón, al volver al colegio tras el verano. Se realizó un grupo de discusión, en el que se utilizaron los mapas corporales ya realizados, para la generación de datos secundarios. Participaron siete niñas y niños, autores de los mapas corporales. Se realizó un análisis temático de la transcripción del grupo de discusión. En este momento se está realizando el análisis de datos, se presentan resultados preliminares. Los mapas corporales como catalizadores de la generación de datos parecen facilitar la identificación de las tensiones entre los discursos de igualdad, expresados claramente por las y los niños, y las discrepancias en el hacer cotidiano u ocupaciones (juegos, vestimenta, roles, etc.) en la infancia, la expresión de género y los estereotipos hegemónicos.
\end{abstract}

Palabras clave: Mapas Corporales Narrados; Género; Terapia Ocupacional; Ciencia de la Ocupación; Infancia; Escuela.

\section{OCCUPATIONAL AND GENDER ANALYSIS IN CHILDHOOD: APPLICATION OF BODY MAP STORYTELLING FROM A DISCUSSION GROUP}

\begin{abstract}
This research aims to explore the perceptions of Primary Education students of a public school in A Coruña (Galicia, Spain), regarding their collective imaginary of occupations, gender expression and hegemonic stereotypes. A secondary study is proposed, using a previous socioeducational experience based on body-mapping storytelling, in which a group of girls and boys had co-created the stories of two fictional characters, Verónica and Antón, when they returned to school after summer. A discussion group was held, in which the already created body maps were used, for a secondary data generation. Seven girls and boys, authors of the body maps, participated. A thematic analysis of the discussion group transcript was performed. Data analysis is being carried out at this point, preliminary results are presented. Body-maps as catalysts for data generation seem to facilitate identifying the tensions between the equality discourses, clearly expressed by children, and the discrepancies in daily activities or occupations (games, clothing, roles, etc.) in childhood, gender expression and hegemonic stereotypes.
\end{abstract}

Keywords: Body Maps Storytelling; Gender; Occupational Therapy; Occupational Science; Childhood; School.

\section{INTRODUCCIÓN}

La Convención sobre los Derechos del Niño (Unicef, 1989) reconoce la capacidad de las niñas y los niños de expresar libremente sus opiniones, por lo tanto es imprescindible que cuenten con voz en asuntos relativos a la infancia. Sin embargo, sus aportaciones suelen ser silenciadas o consideradas como no válidas desde la perspectiva de las personas 
adultas (Vergara, Peña, Chávez, \& Vergara, 2015), sin reconocer su derecho de expresión como sujetos políticos, con capacidad de participación, decisión, transformación y aportación al tejido social.

El proceso de transición ocupacional o cambio de ocupaciones ${ }^{1}$, que se produce en la infancia es constante, siendo clave proporcionar espacios que permitan promover prácticas orientadas a la promoción de una educación para la ciudadanía global "capacitar a los individuos para la reflexión crítica sobre su legado y los procesos culturales, para imaginar soluciones futuras diferentes y para asumir responsabilidades sobre sus decisiones y acciones"(Andreotti, 2014, p.63). Esta investigación se sitúa en la infancia y en las interacciones que se producen o reproducen en los espacios compartidos por niñas y niños, siendo el género el eje y la interseccionalidad que se produce en torno a él -en las edades más tempranas-, lo que puede motivar o limitar la participación. Existe un creciente interés dentro del campo de la Terapia Ocupacional (TO) y Ciencia de la Ocupación (CO) en relación al género y sus implicaciones en diferentes dimensiones, identificándolo como un ámbito de investigación prioritario (Angell, 2014; Carra, 2014), ya que en relación a otras disciplinas próximas como la psicología o sociología, es un campo poco explorado y con escasa literatura reciente dentro de la TO/CO.

Se han realizado investigaciones en TO/CO que abordan cómo el género impacta en diferentes dimensiones de la vida diaria (Goodman, Knotts, \& Jackson, 2007; Huff, Rudman, Magalhães, \& Lawson, 2018; Liedberg, Björk, \& Hensing, 2010; Mansilla- Rivera, AsteteValdebenito, Garrido-Salazar, \& Maldonado-Molina, 2017; Rivas-Quarneti, MovillaFernández, \& Magalhães, 2017; Wada, Backman, \& Forwell, 2010). Sin embargo, son pocas las investigaciones en TO/CO que profundicen en la relación del género y la infancia, pese a lo que se infiere de la evidencia existente: en el estudio realizado por Stagnitti et al. (2010) encontraron que las niñas y los niños de 4-5 años optaban por jugar de forma segregada y escoger material de juego acorde a los roles de género hegemónicos. Angell (2014) muestra la importancia de los contextos y las ocupaciones en la reproducción de injusticias y vulneración de derechos ocupacionales y la interseccionalidad de inequidades relacionadas

\footnotetext{
${ }^{1}$ Se refiere a los desafíos que conllevan los cambios vitales, entendido en la infancia como el proceso de mutación en todos los contextos producido con el paso de los años, como puede ser el avance de curso académico o la transición de la escuela primaria a la secundaria (Asbjørnslett, Engelsrud, \& Helseth, 2015). Similar al que se produce en otras etapas y que tiene un gran impacto en la vida de las personas: la jubilación - fin de la etapa de trabajo remunerado-(Wiseman \& Whiteford, 2009); en un proceso migratorio - cambio de contextos y ocupaciones-(Santiago-Lista, Rivas-Quarneti, Añón-Loureiro, \& Movilla-Fernández, 2014) o en la experiencia de las personas trans —-desde la infancia a la adultez-(Schneider, Page, \& van Nes, 2019).
} 
con el género y la infancia, señalando la importancia de generar evidencia que permita avanzar en su abordaje dentro de la TO/CO. Es importante analizar e investigar las oportunidades culturales y contextuales (Hocking, 2000) que limitan o favorecen la participación ocupacional (Shaw, 2009), ya que son clave para el desarrollo y socialización en la infancia y en consecuencia, determinarán las prácticas de la futura ciudadanía.

\section{OBJETIVOS}

El objetivo general de esta investigación fue explorar las percepciones de alumnado de Segundo Ciclo de Educación Primaria de un colegio público del entorno urbano de A Coruña (Galicia, España), sobre los imaginarios colectivos en relación con las ocupaciones, expresión de género y estereotipos hegemónicos.

\section{METODOLOGÍA}

\subsection{Diseño del Estudio}

Esta investigación parte de un proyecto previo (Tojeiro-Ríos, Rodríguez, \& Rivas-Quarneti, 2019) en el que se emplearon mapas corporales narrados grupales (Gastaldo, RivasQuarneti, \& Magalhães, 2018a) para conocer cómo vivía el alumnado de $2^{\circ}$ de Educación Primaria de un colegio público de la provincia de A Coruña, la transición ocupacional (cambio en las actividades del día a día) al inicio del curso escolar y reflexionar juntos sobre la escuela que quería el alumnado. El mapeo corporal narrado es una metodología de investigación cualitativa crítica (Gastaldo, et al, 2018a), que fue adaptada en esta experiencia socioeducativa, en la que se crean mapas corporales "[imágenes del cuerpo humano a tamaño real] dibujando, pintando o empleando otras técnicas artísticas para representar visualmente aspectos de la vida de las personas, sus cuerpos y el mundo en el que viven. El mapeo corporal es una forma de contar historias, al igual que los tótems que contienen símbolos con significados diferentes, pero cuyo significado sólo puede entenderse en relación a la historia global del creador y su experiencia" (Gastaldo, Magalhães, Carrasco, \& Davy, 2018b, p.5). Así, se utilizaron los mapas corporales narrados con las niñas y niños, a quienes se les preguntó cómo creían que era el día a día de dos personajes ficticios, Verónica y Antón, antes, durante y tras el inicio de curso, así como qué esperaban y cómo les gustaría que fuese la escuela. Estos personajes, vivían en su misma comunidad y tenían su misma edad. Fueron las niñas y niños quienes crearon la historia de estos personajes, utilizando el mapeo corporal (Tojeiro-Ríos et al., 2019). El resultado de esta 
iniciativa dio lugar a una exhibición del material en el que el alumnado compartió con la comunidad universitaria sus impresiones, reivindicando su contribución en un contexto en el que habitualmente no tienen voz (Tojeiro-Ríos et al., 2019). Por lo que en esta experiencia se emplearon los mapas corporales como un instrumento de concienciación/activismo desde una perspectiva socioeducativa, facilitando la expresión artística de ideas y ocupaciones del alumnado y su traslado a la comunidad universitaria, siendo el arte una herramienta que facilitó la expresión y participación cultural. Este estudio, que busca explorar las percepciones de la infancia, sobre los imaginarios colectivos en relación con las ocupaciones, expresión de género y estereotipos hegemónicos, es una continuación de este proyecto, al que se ha incorporado la segunda autora (forma parte de su Trabajo de Fin de Máster). Puesto que los mapas corporales realizados abordaban el objeto de estudio (género y ocupaciones), se propuso emplear dichos mapas como catalizador de la conversación, siendo una herramienta de generación secundaria de datos. Otras investigación han utilizado esta fórmula con éxito. Por ejemplo, Chenhall, Davison, Fitz, Pearse, \& Senior (2013) señalan que los mapas corporales narrados pueden ser utilizados, posteriormente, como una herramienta para realizar una discusión adicional que aborde los contenidos expresados de forma artística, siendo igual de importante la interpretación, que la creación del mapa. Otra forma de explotar el potencial de los mapas corporales narrados es la que realizan Macgregor \& Mills (2011) que reúnen años después a personas participantes en la creación de mapas para conocer como han cambiado sus percepciones con el paso del tiempo. Siguiendo lo que describen estas investigaciones, se planteó realizar un grupo de discusión (GD) con las personas autoras de los mapas corporales, para abordar la pregunta de investigación.

\subsection{Selección de las Personas Participantes}

Las personas que participan se seleccionaron siguiendo un muestreo intencional para alcanzar los objetivos de la investigación. En la fase de diseño de los mapas corporales participaron 30 personas, de las cuales, para esta investigación se estableció un tamaño muestral paritario tentativo de 4-8 personas participantes - teniendo en cuenta para este criterio el conocimiento de la muestra y contexto de estudio de tres investigadoras del equipo-. Por otra parte, en una investigación con diseño metodológico similar, contaron con 5 personas participantes (Macgregor \& Mills, 2011), considerándolo como un grupo suficiente para alcanzar los objetivos de investigación propuestos, lo que también sirve para orientar el tamaño muestral de este estudio. El interés central de esta investigación fue la 
comprensión de la información proporcionada en profundidad y el análisis de su complejidad, riqueza y diversidad desde una perspectiva situada, crítica y contextual. Por lo tanto, se justifica el tamaño muestral en base a la posibilidad de trasmitir algo relevante y significativo sobre el fenómeno de estudio (Mayan, 2009)..

Las características que determinan la participación en el estudio se establecen siguiendo unos criterios de inclusión-exclusión, que determinan el cribado, siendo los siguientes:

Tabla 1. Criterios de inclusión en el estudio de las personas participantes

\begin{tabular}{l|l}
\hline Criterios de inclusión & Justificación \\
\hline $\begin{array}{l}\text { Alumnado de Segundo Ciclo de Educación Primaria (8-10 } \\
\text { años) que haya participado en la co-creación de los mapas } \\
\text { corporales narrados de Verónica y Antón }\end{array}$ & Conocedores de la realidad de estudio \\
\hline $\begin{array}{l}\text { Firma del consentimiento informado por parte de los } \\
\text { responsables legales del o la participante }\end{array}$ & $\begin{array}{l}\text { Necesario para cumplir la normativa jurídica y } \\
\text { ética }\end{array}$ \\
\hline Consentimiento del participante & Reconocer su validez como sujeto autónomo \\
\hline
\end{tabular}

\subsection{Aspectos Éticos}

EI GD se basó en el intercambio de opiniones en base a las percepciones sobre los mapas corporales de Verónica y Antón, cuyas historias habían sido creadas por ellos/as mismas, por lo que las personas participantes pudieron expresar sus opiniones de forma significativa y situada. Asimismo, como en la creación de los mapas corporales se usaron dos personajes ficticios, se evitó hablar de sí mismas, favoreciendo un mecanismo de protección, ya que se trata de una situación menos incómoda o estresante para exponer en grupo (Chenhall et al., 2013; Glantz, Halperin, \& Hunt, 1998). La información generada fue tratada de forma confidencial, respetando el anonimato y eliminando datos que pudieran conducir a la identificación de las personas participantes. Se solicitó la valoración de la investigación al Comité de Ética de la Investigación y la Docencia de la Universidad de A Coruña, emitiendo un informe favorable, considerando que el estudio cumplía de forma suficiente los aspectos éticos y jurídicos relevantes para la investigación.

\subsection{Proceso de Entrada al Campo}

Se propuso la participación en un GD a las personas que crearon los mapas corporales narrados, mediante la mediación de parte del equipo investigador. Fueron las personas encargadas de actuar como intermediadoras para realizar los trámites burocráticos necesarios. Explicaron al grupo en qué consistía la investigación y proporcionaron 
documentos informativos y de consentimiento informado, que también se remitieron por email a las personas representantes legales de las personas participantes.

Se acordó el día y el lugar del GD atendiendo a las necesidades de las familias y a las consideraciones éticas, citándolas en un espacio neutro —un aula de la Facultad de Ciencias de la Salud de la Universidad de A Coruña, a la que se habían desplazado previamente durante la exhibición de los mapas corporales a la comunidad universitaria-. Tuvo lugar en un momento en el que no había actividad educativa (fin de semana), favoreciendo de este modo la preservación del anonimato.

\subsection{Método de Recogida de Datos}

La fuente de generación de datos fue el GD, en el que participaron siete personas —un año y siete meses después de la creación de los mapas corporales, favoreciendo un tiempo de distanciamiento que favorecería la reflexión sobre sus personajes-, que compartieron de forma grupal ideas y reflexiones que surgieron, sobre las cuestiones que se fueron planteando, radicando la riqueza en el proceso y debate creado. Se ofreció la oportunidad a las madres, padres o tutores legales de las personas participantes, de estar presentes como personas observadoras externas al GD ${ }^{2}$-aunque finalmente decidieron, de forma individual, no estar en la sala-.

El grupo contó con la participación de tres facilitadoras del equipo investigador que guiaron la discusión, orientando el debate hacia preguntas que generaron opiniones y mediaron para crear un ambiente distendido. La primera autora, parte del trabajo original y persona conocida por el alumnado por su participación en distintos proyectos participativos en su escuela, fue la figura encargada de generar continuidad con la creación de los mapas corporales y confianza para con las otras dos investigadoras. Se siguió un guion con preguntas estructuradas en torno a los objetivos del estudio para facilitar el abordaje de las cuestiones clave para la investigación. Por ejemplo: "Si yo fuera una extraterrestre que acaba de llegar a este planeta, me podrías explicar cuál es la diferencia entre Verónica y Antón” (...)” ¿A qué juegan en el recreo?”, “¿Cuál es la asignatura que más les gusta? ¿Y la que menos?”, “¿Cuál es su deporte favorito?”, etc.

\footnotetext{
${ }^{2}$ Su presencia se orientó al ejercicio de los derechos de revocación de consentimiento de participación, contando en este caso, con el requerimiento ético de confidencialidad referente al contenido abordado en el grupo.
} 
La discusión tuvo una extensión de una hora y media, documentada empleando grabación en audio -con la conformidad de los requerimientos éticos, que se explicitan en los documentos de información proporcionados a las personas implicadas en la investigación-, con el objetivo de ser empleada para facilitar el análisis de los datos que dan forma al cuerpo del estudio.

\subsection{Análisis de los Datos}

Para examinar los datos orales se optó por un análisis temático, definido como "un método de análisis cualitativo de datos para identificar, analizar, interpretar e informar patrones (temas) a partir de datos cualitativos" (Braun \& Clarke, 2006, p.8). Se siguieron las siguientes fases (Braun \& Clarke, 2006, p.35): familiarización con los datos, generación de categorías o códigos iniciales, búsqueda de temas, revisión de temas, definición y denominación de temas, producción del informe final. Para facilitar la profundización en los datos se realizó un mapa temático, que ayudó a componer las piezas y dibujar una historia. En este proceso, para la gestión e interpretación de la información, se utilizó el software ATLAS.ti (versión 8).

\section{RESULTADOS}

La investigación se encuentra en la fase de análisis de los datos, por lo que se presentan los resultados preliminares, que permiten una aproximación inicial al objetivo de investigación.

Los/as niñas contextualizan a los personajes en una escuela como un espacio positivo de aprendizaje y de relación social con iguales, siendo visto su colegio ideal como un lugar lleno de vida. El juego que parece predominar en el patio "correr [...] fútbol [...]" (extracto del GD) sugiere que se realiza de forma segregada "[Antón juega] con sus amigos [...] tampoco le importa jugar con las niñas..." (extracto del GD), aunque se vislumbran espacios de juego compartidos "normalmente juega con chicos y chicas y juegan al pilla pilla..." (extracto del GD). En las ocupaciones de ocio relacionadas con los videojuegos identifican ciertos sesgos que pueden limitar la participación en igualdad "en la portada del FIFA [videojuego de fútbol] todo son chicos" (extracto del GD). Así como en la música "las chicas siempre tienen que estar bailando en sus canciones" (extracto del GD), que no parecen identificarse con el rol asignado en este espacio. 
Perciben ciertas prendas del vestido como elementos para la expresión de género "aquí si un chico le da por ponerse una falda... [...] nadie se lo va a prohibir" (extracto del GD), aunque surgen opiniones diversas "no existen bañadores de niño" (extracto del GD), que contrastan en relación con las expectativas sociales, diluyendo el binomio que parece predominar en la categorización de la ropa. Existe cierta confusión con la expresión de género en sus múltiples formas "un chico que era, que le gustaba ser chica entonces él, eh...era chica [...] o sea que se hizo un cambio de sexo" (extracto del GD), por lo que la inclusión de corporalidades no normativas que permitan cuestionar lo establecido y sirvan de referentes podría ser positivo como estrategia para concienciar sobre la igualdad en sus diversas formas.

Aunque su discurso pretende transmitir que los estereotipos son algo del pasado "yo creo que antes, los niños no podrían estar con las niñas...pero...ahora sí y no pasa nada" (extracto del GD), se identificaron tensiones "le va a decir a la señora yo quiero unos zapatos [...] a un zapatero que haga zapatos" (extracto del GD). El imaginario de la organización social patriarcal en torno al trabajo reproductivo y productivo parece resonar con cierta sutileza.

\section{DISCUSIÓN Y CONCLUSIONES}

La metodología empleada, utilizando los mapas corporales narrados realizados con anterioridad, como catalizadores de la discusión, permitieron a las personas participantes en el GD expresar sus percepciones en relación con las ocupaciones en la infancia, la expresión de género y los estereotipos hegemónicos, evidenciando que es necesario realizar más esfuerzos en estas áreas, para progresar hacia un alumnado más consciente de los desafíos en materia de igualdad.

En esta investigación emergen nuevos retos ligados a la metodología, reconociendo como clave la búsqueda de métodos guiados por la imaginación, la ética, la adaptación contextual y significativa (Johnson, Hart, Colwell, West, \& Carvalho, 2014)—uso de videos, conchas o arena, impresoras 3D, plastilina - que impliquen la participación activa de niñas y niños, como requisito para desarrollar una evidencia situada y transformadora. Hasta el momento, este estudio parece visibilizar como las prácticas diarias en educación, política y cultura deben ir encaminadas a fomentar el pensamiento crítico de niñas y niños para de este modo crear una ciudadanía más inclusiva. Abriendo, así, posibilidades a métodos participativos innovadores y a la creación de nuevos discursos e imaginarios. 
Agradecimientos. Esta investigación ha sido posible gracias a la colaboración de niñas y niños y sus familias, a quienes debemos un gran reconocimiento por su compromiso.

\section{REFERENCIAS}

Andreotti, V. (2014). Educação para a Cidadania Global - Soft versus Critical. Sinergias - diálogos educativos para a transformação social, (1), 57-65. Recuperado de http://www.sinergiased.org/index.php/revista/item/53-vanessa-andreotti-educacao-para-a-cidadania-globalsoft-versus-critical

Angell, A. M. (2014). Occupation-centered analysis of social difference: Contributions to a socially responsive occupational science. Journal of Occupational Science, 21(2), 104-116. https://doi.org/10.1080/14427591.2012.711230

Asbjørnslett, M., Engelsrud, G. H., \& Helseth, S. (2015). How Children with Disabilities Engage in Occupations during a Transitional Phase. Journal of Occupational Science, 22(3), 320-333. https://doi.org/10.1080/14427591.2014.952365

Braun, V., \& Clarke, V. (2006). Using thematic analysis in psychology. Qualitative Research in Psychology, 3(2), 77-101. https://doi.org/10.1191/1478088706qp063oa

Carra, K. A. (2014). Gender, disaster, and occupational health. Australian Occupational Therapy Journal, 61(1), 32-32. https://doi.org/10.1111/1440-1630.12078

Chenhall, R., Davison, B., Fitz, J., Pearse, T., \& Senior, K. (2013). Engaging Youth in Sexual Health Research: Refining a "Youth Friendly" Method in the Northern Territory, Australia. Visual Anthropology Review, 29(2), 123-132. https://doi.org/10.1111/var.12009

Gastaldo, D., Magalhães, L., Carrasco, C., \& Davy, C. (2018b). Mapas corporales narrados como investigación. Consideraciones metodológicas para contar las historias de trabajadores indocumentados a través del mapeo corporal (Trad. N. Rivas-Quarneti, S. Sánchez-Pérez, V. Vinzón y S. Veiga-Seijo).

Gastaldo, D., Rivas-Quarneti, N., \& Magalhães, L. (2018a). Body-Map Storytelling as a Health Research Methodology: Blurred Lines Creating Clear Pictures. Forum: Qualitative Social Research, 19(2), 43-68. https://doi.org/10.17169/FQS-19.2.2858

Goodman, J., Knotts, G., \& Jackson, J. (2007). Doing dress and the construction of women's gender identity. Journal of Occupational Science, 14(2), 100-107. https://doi.org/10.1080/14427591.2007.9686590

Hocking, C. (2000). Having and using objects in the western world. Journal of Occupational Science, 7(3), 148157. https://doi.org/10.1080/14427591.2000.9686478

Huff, S., Rudman, D. L., Magalhães, L., \& Lawson, E. (2018). 'Africana womanism': Implications for transformative scholarship in occupational science. Journal of Occupational Science, 25(4), 554-565. https://doi.org/10.1080/14427591.2018.1493614

Johnson, V., Hart, R., Colwell, J., West, A., \& Carvalho, X. (2014). International Innovative Methods for Engaging Young Children in Research. En Methodological Approaches (pp. 335-356). University of Brighton. https://doi.org/10.1007/978-981-287-020-9_18

Liedberg, G. M., Björk, M., \& Hensing, G. (2010). Occupational therapists' perceptions of gender - A focus group study. Australian Occupational Therapy Journal, 57(5), 331-338. https://doi.org/10.1111/j.14401630.2010.00856.x

Macgregor, H., \& Mills, E. (2011). Framing rights and responsibilities: accounts of women with a history of AIDS activism. BMC International Health and Human Rights, 11(Suppl 3), 12 . Recuperado de https://bmcinthealthhumrights.biomedcentral.com/articles/10.1186/1472-698X-11-S3-S7\%0D 
Mansilla- Rivera, M. I., Astete-Valdebenito, N. C., Garrido-Salazar, L. C., \& Maldonado-Molina, F. C. (2017). Terapeutas ocupacionales: influencia del género en su desempeño laboral. Revista Chilena de Terapia Ocupacional, 17(1), 155. https://doi.org/10.5354/0719-5346.2017.46388

Rivas-Quarneti, N., Movilla-Fernández, M. J., \& Magalhães, L. (2017). Immigrant women's occupational struggles during the socioeconomic crisis in Spain: Broadening occupational justice conceptualization. Journal of Occupational Science, 7591(August), 1-13. https://doi.org/10.1080/14427591.2017.1366355

Santiago-Lista, C., Rivas-Quarneti, N., Añón-Loureiro, L., \& Movilla-Fernández, M.-J. (2014). Relatos de transición ocupacional de mujeres inmigrantes en España. TOG (A Coruña), 11(20), 1-19. Recuperado de http://www.revistatog.com/num20/pdfs/original5.pdf

Schneider, J., Page, J., \& van Nes, F. (2019). "Now I feel much better than in my previous life": Narratives of occupational transitions in young transgender adults. Journal of Occupational Science, 26(2), 219-232. https://doi.org/10.1080/14427591.2018.1550726

Shaw, L. (2009). Reflections on the importance of place to the participation of women in new occupations. Journal of Occupational Science, 16(1), 56-60. https://doi.org/10.1080/14427591.2009.9686643

Stagnitti, K., Rodger, S., \& Clarke, J. (2010). Determining gender-neutral toys for assessment of preschool children's imaginative play. Australian Occupational Therapy Journal, 44(3), 119-131. https://doi.org/10.1111/j.1440-1630.1997.tb00764.x

Tojeiro-Ríos, A., Rodríguez, N., \& Rivas-Quarneti, N. (2019). Estudio crítico de la escuela desde la mirada de I@s niñ@s: los mapas corporales narrados de Verónica y Antón. En I Congreso Internacional de Educación e Intervención: Psicoeducativa, Familiar y Social (p. 248). Las Palmas de Gran Canaria.

Unicef. (1989). Convención sobre los derechos del niño. Estado Mundial de la Infancia 1991. Madrid. https://doi.org/10.18356/51f8034c-es

Vergara, A., Peña, M., Chávez, P., \& Vergara, E. (2015). Los niños como sujetos sociales: El aporte de los Nuevos Estudios Sociales de la infancia y el Análisis Crítico del Discurso. Psicoperspectivas. Individuo y Sociedad, 14(1), 55-65. https://doi.org/10.5027/psicoperspectivas-Vol14-Issue1-fulltext-544

Wada, M., Backman, C. L., \& Forwell, S. J. (2010). Theoretical perspectives of balance and the influence of gender ideologies. Journal of Occupational Science, 17(2), 92-103. https://doi.org/10.1080/14427591.2010.9686680

Wiseman, L., \& Whiteford, G. (2009). Understanding occupational transitions: A study of older rural men's retirement experiences. Journal of Occupational Science, 16(2), 104-109. https://doi.org/10.1080/14427591.2009.9686649 